\title{
La ineptitud del digital academic: precariedad y salud en el mundo universitario
}

\author{
Inadequacy of a digital academic: \\ precariousness and health in the University
}

FRANCESCA COIN (Università Ca' Foscari)

Artículo recibido: 28 de junio de 2018

Solicitud de revisión: 26 de noviembre de 2018

Artículo aceptado: 04 de febrero de 2019

Coin, Francesca (2019). La ineptitud del digital academic: precariedad y salud en el mundo universitario. Recerca. Revista de Pensament i Análisi, 24(1), pp. 114-133.

\section{Resumen:}

Inspirado por el trabajo de Deborah Lupton, Inger Mewburn y Pat Thomson, The Digital Academic: Critical Perspectives on Digital Technologies in Higher Education (2017), este artículo examina el yo digital académico contemporáneo. Más allá de la utilidad que pueden tener en cuanto a la interacción, plataformas digitales como Academia.edu, Linkedin, Google Scholar, etc. transforman al académico o académica en un sujeto digital cuyo rendimiento se controla constantemente hasta convertirlo en prisionero de una creciente dataveillance, una vigilancia a través de los datos. En este contexto, analizamos el impacto de los mencionados procesos de monitorización continua del rendimiento y la productividad sobre las condiciones de vida y trabajo de los profesionales universitarios contemporáneos, en un momento en el que las tensiones, los conflictos y el malestar que habitan la universidad neoliberal parecen volverse cada vez más incapacitantes.

Palabras clave: nueva gestión pública, precariedad, salud mental, tecnología digital, universidad neoliberal.

\section{Abstract}

Drawing inspiration from the work of Deborah Lupton, Inger Mewburn and Pat Thomson, The Digital Academic: Critical Perspectives on Digital Technologies in Higher Education (2017), this paper looks at the digital self of contemporary academics. Using digital platforms such as Academia.edu, Linkedin, Googlescholar, digital academics can increase their daily interactions and the impact of their research. More than being interaction tools, these tools transform the academics into digital individuals whose performance is constantly monitored until they find themselves prisoner of a growing «dataveillance» (Lupton, Mewburn and 
Thomson, 2017). In this context, the question concerns not only the impact of digital technologies on the quality of contemporary research, but also the impact of digital surveillance on the living and working conditions of contemporary academics, at a time where the tensions, conflicts and malaise that inhabit the neoliberal university have become ever more manifest.

Key Words: new public management, academic precarity, mental health, digital technologies, neoliberal university.

\section{LAS DIMENSIONES AFECTIVAS DE LA ACADEMIA DIGITAL}

El 22 de agosto de 2017 el Times of Higher Education publicó un artículo con el expresivo título de «Academics face higher mental health risk than other professions» (Else, 2017). Desde las primeras líneas, se revelaba como un artículo discordante respecto a la narrativa dominante del trabajo académico (definido, muchas veces, como una ocupación privilegiada, realizada por personas bien pagadas que viven en una torre de marfil). En contraste con esta narrativa, el artículo se detenía en la investigación de Susan Guthrie, Catherine Lichten, Janna van Belle, Sarah Ball, Anna Knack y Joanna Holman encargada por la Royal Society y el Wellcome Trust para evaluar el estado de salud mental de los investigadores y académicos del Reino Unido (Guthrie et al., 2017). La investigación realizaba una revisión exhaustiva de la literatura que en los últimos años se ha ocupado de los cambios en la salud mental en las universidades. Según los resultados presentados, la mayoría de los investigadores universitarios consideraban su trabajo como estresante. El personal académico informaba de niveles de burnout más altos que en el resto de las profesiones. No solo eso, sino que estos niveles de agotamiento alcanzados resultaban equiparables al de los profesionales de sectores de alto riesgo de burnout como, por ejemplo, la sanidad. Igualmente, la investigación enfatizaba que el número de estudiantes de doctorado y personal académico que ha desarrollado un problema mental es proporcionalmente mayor que en el resto de la población. Según el informe, una elevada cantidad de estudiantes de doctorado (más del $40 \%$ ) reportaban síntomas de depresión, problemas emocionales o altos niveles de estrés (Guthrie et al., 2017: 15).

Entre los factores determinantes de esta situación, la investigación cita el género, lo que significa que las mujeres sufren una mayor exposición al estrés que los hombres y una mayor dificultad para conciliar el trabajo y la vida familiar. Otro factor destacado por los autores es la personalidad académica, 
que indica que la actitud hacia la autocrítica hace que los académicos sean más susceptibles de padecer estrés y, a veces, más inclinados a expresarlo. La investigación cita un artículo aparecido en el New York Times (Scelfo, 2015) donde se traza una conexión entre la «cultura de la perfección» y el crecimiento de los casos de suicidio de los estudiantes universitarios, tanto entre estos como entre el personal académico, y se evidencia la presencia de una línea muy sutil entre la tendencia existente al hiperrendimiento y la angustia del fracaso. En muchos casos, el mero hecho de cometer un error en una prueba de admisión o en un examen llevó a los jóvenes estudiantes a concluir que estaban «fallando», en lugar de pensar, simplemente, que habían errado una meta parcial, señaló Scelfo (2015). Según esta autora, podemos hablar de la cultura de la perfección siempre que la impresión de tener que llegar a la excelencia se traduzca en el sentimiento de estados mentales de «desaliento, alienación, ansiedad o depresión». En estos casos, la culpabilización se traduce en la sensación de «ser defectuoso» o, por decirlo de otra manera, no ser lo suficientemente bueno. «No se trata de haber tenido un mal desempeño — continúa Scelfo-, se trata de no ser bueno para nada».

Hace algún tiempo, Mark Fisher (2014) escribió un artículo apasionante llamado precisamente «Good for nothing» ('Bueno para nada'). En ese artículo, Fisher describió la relación entre la depresión, la percepción de inadecuación y la lucha competitiva neoliberal. A diferencia de las ideas dominantes en la psiquiatría, que identifican los orígenes de la depresión «en el mal funcionamiento de la química del cerebro, que ha de ser tratada con productos farmacéuticos» y al contrario que el psicoanálisis, que investiga sus raíces en el entorno familiar, para Mark Fisher la causa de la depresión es ese tipo de «inferioridad ontológica» en la que la sociedad democrática esconde la existencia de un proyecto de resubordinación de la sociedad. Para Fisher, la voz interior que le ha acompañado personalmente a lo largo de su vida, y que le repetía «no eres bueno para nada», es una voz que nace de la violencia con la que la sociedad neoliberal decide expulsar del mercado y de la protección social a todos aquellos que son juzgados incapaces de aportar un valor agregado. «No eres bueno para nada» es una voz que nace del empeño por negar la percepción de la violencia de clase.

El propósito de este texto es comenzar a explorar el concepto de ineptitud, de sentirse inadecuado. Partimos de la idea de que la ineptitud conlleva la introyección y, por lo tanto, la legitimación del enfoque a través del cual el neoliberalismo expulsa del mercado a porciones enteras de la sociedad. La universidad encarna gran parte de la responsabilidad de la expulsión actual y 
la ha encarnado en momentos anteriores. Por decirlo con palabras de Kenneth J. Arrow (1973), cuando en la década de 1970 se alcanzaron situaciones de pleno empleo, fue la educación universitaria la que se encargó de actuar como un filtro social. Para Arrow, no es una casualidad que la universidad sea un filtro, un dispositivo de criba que, en sus duras palabras, «no contribuye de ninguna manera al rendimiento económico, ni aumenta las habilidades cognitivas o la socialización», sino que «sirve como un dispositivo para la selección: ordena a los individuos» según sus capacidades y ofrece esta información a los empresarios» (Arrow, 1973: 195). En términos semejantes, Kenneth Burdett (1978) explica que no se trata solo de catalogar, clasificar y presentar el capital humano, sino de filtrarlo: se busca clasificar y filtrar a estudiantes e investigadores (entendidos como capital humano); clasificar y filtrar el conocimiento (investigación aplicada preferible a la investigación básica) y, por último, clasificar y filtrar estructuras (universidades o departamentos), con la finalidad de asignar recursos solo a las unidades de producción más competitivas de cara al mercado. Desde entonces, los profesores y los estudiantes, el conocimiento y las estructuras están en el centro de una cuantificación, medición, evaluación y selección basada en la monitorización constante de su desempeño para establecer una clasificación de aquellos recursos productivos que pueden generar mayor valor añadido. En este contexto, la ineptitud dentro del digital academic, como condición afectiva de la universidad digital, no deriva, en palabras de Mark Fisher, de una «inferioridad ontológica» de las personas, sino de la violencia inherente a la decisión política de filtrar a los sujetos elegibles para obtener mayor valor económico y social.

La excelencia y el fracaso, en esta perspectiva, no son consecuencias de la conducta individual, sino el resultado estructural de una política que hace de la organización jerárquica un instrumento de expulsión clave en la política contemporánea. Un instrumento, además, en oposición con los valores de la cultura democrática y que presenta la exclusión como una consecuencia de la incompetencia de los miembros de la sociedad. Sean estos estudiantes que no alcanzan una nota suficiente o investigadores precarios que no pueden encontrar un puesto estable o fondos para proyectos de investigación, en la interpretación mercantilista estos no son ejemplos de expulsiones sociales, a la manera de Saskia Sassen (2015), sino de incompetencias, de proyectos insuficientes, de estudiantes buenos para nada o de conocimiento poco valorizable. La insuficiencia, en este sentido, es una especie de estado de nervios, que produce una especie de desgarro en el mundo académico contemporáneo que, por un lado, se mira continuamente a través de los ojos del mercado y, por otro, es 
rehén de la violenta capacidad de expulsión que este provoca y cuyos efectos son desconocidos. Retomo el concepto de estado de nervios del viejo libro de Tsitsi Dangarembga (1988) según el cual los individuos se encuentran a veces prisioneros de dos regímenes diferentes de verdad, entre ellos contradictorios y que deben ser descifrados para interpretarse a sí mismos y actuar en el mundo que los rodea. La pregunta es ¿cómo coexisten estos valores contradictorios en el mundo académico digital? ¿De qué manera se sufre y de qué manera se gestiona la lucha competitiva por la supervivencia? En definitiva, ¿es posible transformar la supuesta ineptitud individual del mundo académico digital en una especie de indignación que reaccione contra el proceso de expulsión en el que la universidad neoliberal actúa decididamente como un filtro social?

\section{LA MIRADA NEUTRA DE LA JERARQUÍA}

La década de 1980 fue un momento clave en el que comenzó un replanteamiento del papel de la universidad en relación con el mercado laboral. El problema radicaba, en aquellos años, en la progresiva presencia en el mercado laboral de dinámicas de polarización entre MacJobs y McJobs y los respectivos niveles de cualificación que reclamaban. En opinión de los economistas David H. Autor, Lawrence F. Katz y Melissa S. Kearney, la evolución del trabajo (a partir de la década de 1990) estuvo marcada por una polarización, con un fuerte crecimiento de empleos altamente cualificados, un débil aumento de aquellos con cualificaciones intermedias y un incremento modesto en trabajos poco cualificados (Hirtt, 2010). En este contexto:

El problema que se planteaba a los responsables de la gestión del sector educativo es qué hacer con el modelo que se había consolidado entre las décadas de 1950 y 1980 caracterizado por sistemas escolares masificados, con largos recorridos escolares de formación estándar. Históricamente, esto se había integrado bien en momentos de confianza en un capitalismo próspero, con un crecimiento económico fuerte y duradero, que demandaba un aumento continuo en los niveles de capacitación. Sin embargo, el periodo que se abrió tras la crisis de 1973, sumado a los mencionados cambios en la polarización de las cualificaciones, plantearon un interrogante sobre el sistema educativo acerca de cuál podría ser la base formativa común para los futuros ingenieros, por un lado, y los futuros trabajadores descualificados, por el otro (Hirtt, 2010).

Desde entonces, el sistema educativo, y en particular el nivel universitario, fue profundamente repensado asumiendo el mercado como auténtico principio de realidad en la acción de gobierno. La intención, según se mostró en 
numerosos informes de la OCDE y de otros organismos supranacionales como la UE, el FMI o el Banco Mundial, fue la de introducir reformas de tipo businesslike para convertir el sector público de la enseñanza en un área permeable al mercado. Así comenzó, en particular en el ámbito anglosajón, un proceso de reforma de la educación pública inspirado por las ideas del New Public Management, con un típico paquete de reformas que incluye:

Cambios en la gobernanza de las universidades; crecimiento de los precios de matrícula (a menudo acompañado de una reducción de becas y otros derechos a la educación pública); aumento del protagonismo de las instituciones privadas en las políticas educativas y científicas; introducción de la competencia a la hora de acceder a financiación estatal, y output modeling (Marginson, 2009: 4).

Este modelo basado en resultados (output) tiene como objetivo garantizar que todas las actividades del complejo mundo de la investigación y la educación se conviertan en productos cuasi mercantiles, productos con su propio mercado. En este contexto, la idea de medir el output en lugar del input muestra «un cambio significativo dentro de los sistemas educativos: el enfoque ya no estará en qué se enseña, sino en qué se aprende» (Kostoris Padoa Schioppa, 2012: 5).

En este sentido, la medida de lo que se aprende y lo que se produce permite cuantificar la preparación de los estudiantes y, a su vez, sirve para medir la eficiencia de los docentes y las escuelas (piénsese en la ley americana No Child Left Behind o en la propuesta de los test TeCo). En este sentido, la docencia se subdivide en objetivos operacionables, taxonomizables y cuantificables, para garantizar que la universidad pueda generar habilidades capaces de cumplir los objetivos de los stakeholders y reducir el mismatch con el mercado laboral. De manera similar, la investigación se reorienta de manera que la financiación se dirija a la producción de conocimiento y capital humano útil para el mercado.

\section{LA UNIVERSIDAD-UBER}

De hecho, todas las reformas recientes de los sistemas educativos, desde la escuela a la universidad, desde el Proceso de Bolonia a la Race to the Top de Obama, nacen bajo la inspiración de este paradigma. Retomando el vocabula- 
rio del modelo Just-in-Time, se trató de repensar la cadena de suministro educativo desde el nivel inicial hasta el final, reduciendo el desperdicio relacionado con inversiones no rentables, como podrían ser los casos de sobrecualificación o de mismatch, o la presencia de un capital humano demasiado educado en comparación con las necesidades del mercado. Los conceptos de rendición de cuentas, evaluación, competencia o taylorización de la enseñanza nacen en este marco con la intención de incluir la educación dentro de un esquema neoclásico de costos y beneficios, prometiendo un papel central en el crecimiento económico y la competitividad internacional. Gary Hall describe las consecuencias de este proceso en su libro Uberfication of the university (Hall, 2016), un proceso que fue minando las prácticas colaborativas e imponiendo un individualismo radical basado en la autopromoción y la marca personal.

En este contexto, la investigación y la enseñanza se han transformado profundamente. Desde el punto de vista de la enseñanza, las evaluaciones basadas en el output propician una especie de gran vuelco: los individuos no son ya los que crean el mercado sobre la base de sus necesidades, sino que es el mercado el que los produce. En el Proceso de Bolonia, por ejemplo, los outputs son establecidos por los descriptores de Dublín, que indican las habilidades que los estudiantes deben aprender en un cierto ciclo de estudios, mensurables de forma estandarizada. Más actualmente, en la Universidad-Uber, los estudiantes se ven obligados a moverse como riders, con entregas cada vez más rápidas y fechas de vencimiento que obligan a un control continuo de los esfuerzos dedicados a cada actividad curricular y extracurricular. En este marco, la monitorización del alumno es cada vez más intensa, tanto en la forma de medición de los resultados, outputs, como en el incentivo para usar nuevas tecnologías como medio de autopromoción. En los últimos años, varios interesantes estudios se han centrado en las implicaciones afectivas de la academia digital (Lupton, Mewburn y Thomson, 2017; Hall, 2016; Moore, 2017). El panorama que resulta de este cambio gradual es la transición del sector educativo hacia una economía reputacional, donde el objetivo determinante de cada individuo/unidad productiva parece ser alcanzar posiciones de cabeza en rankings. Independientemente de cómo se mida el rendimiento, un estudiante que quisiera conseguir un trabajo excelente ganaría posibilidades en caso de acceder a las escuelas que se encuentran en las posiciones superiores de los rankings internacionales. Es justo lo que sugirió Kenneth Arrow, la universidad no tiene la obligación de aumentar las habilidades cognitivas, sino que debe, senci- 
1lamente, operar como un filtro social. En un mismo sentido, Roger Abravanel (2008) ha sugerido que el sistema universitario:

Debe tener dos grandes objetivos, crear un pequeño grupo de universidades excelentes a nivel nacional [...] y garantizar el acceso a los mejores y más rentables puestos de trabajo a quienes consigan los títulos en ellas (Abravanel, 2008: 135).

Las nuevas plataformas digitales de Silicon Valley han penetrado, precisamente, en el espacio entre la universidad y el mundo del trabajo para utilizar los big data con el fin de dirigir a los estudiantes a las instituciones más competitivas en el segmento del mercado de trabajo donde mayor es la demanda. El uso de las redes sociales se está convirtiendo, así, en una necesidad para responder a las exigencias del mercado. Para tener un futuro en el mundo profesional, cada uno debe diseñar un business plan que permita afrontar la búsqueda profesional de la manera más eficiente. Así, no basta con tener únicamente una nota media muy alta, sino también confiar en plataformas como Summit Public Schools, Schoolzilla, Basecamp, que tienen como objetivo reducir la distancia que media entre la oferta y la demanda y monitorizar continuamente el desempeño de los aspirantes. Con este fin, Summit Public Schools ha construido una plataforma de aprendizaje personal (PLP) a través de la cual los estudiantes pueden controlar su capacidad de hacer «content delivery» ('entregas de contenido', precisamente como los riders), orientando su capacitación a las demandas del mercado.

La reorientación del saber hacia los intereses del mercado no solo ha transformado la enseñanza, sino también la investigación. En esta parcela, la econometría y la bibliometría se han desarrollado como verdaderos campos de saber capaces de medir el rendimiento individual. La bibliometría, que buscaba catalogar e indexar las publicaciones, acabó ligada al concepto de evaluación, entendido como un procedimiento estándar para valorar la productividad de una actividad o un individuo. Desde 1955, Eugene Garfield buscó hacer más eficiente el proceso de investigación mediante la creación del Science Citation Index, que sistematizó en 1964 en un trabajo de cinco volúmenes para indexar 613 revistas y 1,4 millones de citas (Coin, 2012). En este contexto, Garfield desarrolló software capaz de generar mapas cronológicos de colecciones bibliográficas indexadas por palabras clave, institución, autor, revista, dentro de la Web of Science de ISA y en 1966 definió la historiografía algorítmica como una herramienta que permitía la «recuperación y descubrimiento de información». En la actualidad, este índice está integrado en Web 
of Science, base de datos central de Web of Knowledge. Como es bien sabido, durante largo tiempo Garfield apoyó la necesidad de este trabajo de indexación simplemente para crear redes entre académicos y permitir una rápida difusión de los resultados de la investigación. Estamos hablando, en este sentido, de una tecnología destinada en principio a aumentar la libre circulación de ideas, en lugar de evaluar su valor. El propio Garfield (2006) criticó otros usos de estas tecnologías que no fueran en el sentido que él proponía. Pero, a pesar de esta finalidad inicial de difundir resultados y metodologías de investigación, los indicadores bibliométricos se han acabado convirtiendo con el paso del tiempo en un fin en sí mismos y son utilizados para juzgar el impacto de las revistas científicas y de los artículos que contienen. A pesar de las fuertes críticas, el uso de indicadores bibliométricos se usa hoy para la evaluación de la producción de individuos y estructuras.

Ted Striphas (2011) lo llama cultura algorítmica; con ello se refiere a la clasificación, jerarquización y control de personas, lugares, objetos e ideas. Al mismo tiempo, las tecnologías digitales se han deslizado literalmente por todos los rincones de la vida académica. En esta atmósfera de evaluación, escriben Deborah Lupton, Inger Mewburn y Pat Thomson (2017), se incita a los académicos a utilizar tecnologías digitales como online learning, student feedback y los sistemas automáticos de citas para contribuir al conocimiento y mejorar la capacitación de los futuros trabajadores del conocimiento. Además, la mayoría de las revistas académicas se han digitalizado, lo que permite que las citas puedan cuantificarse y medirse automáticamente con herramientas como Google Scholar, Web of Science o Scopus. Muchas revistas han introducido herramientas de monitorización digital capaces de cuantificar la descarga de artículos, la lectura, los debates en las redes sociales y a veces la comparación con puntuaciones altmetric ('métricas alternativas') de artículos para conocer cuánta atención recibieron en las redes sociales. Los editores también alientan a los académicos a utilizar herramientas como la publicación en open access, así como blogs y redes sociales para visibilizar y promocionar su investigación y facilitar el acceso y la difusión de conocimiento (Lupton, Mewburn y Thomson, 2017: 3).

En resumen, la revolución digital se ha utilizado para clasificar la productividad de cada unidad de producción en un intento de cuantificar su impacto y de ofrecer a los stakeholders una lista de los posibles receptores de financiación capaces de garantizar un retorno de la inversión gracias a su mayor potencial. 


\section{UNA DISLOCACIÓN INTERIOR}

En este contexto, para aumentar sus posibilidades de impacto, se alienta a los docentes a utilizar plataformas digitales como Academia.edu, Linkedin, Googlescholar, e-repositorios universitarios, SlideShare, herramientas de agregación de contenido, a través de las cuales aumentar sus interacciones y el impacto de su investigación. El sujeto académico cuantificado, que Lupton (2016) define como «academic quantified self», es impulsado, o mejor inducido, a recopilar continuamente datos sobre sus actividades de investigación y docencia y a utilizar el análisis de los mismos para mejorar su desempeño. Como mínimo, el investigador es rápidamente presa del síndrome de publish or perish, un síndrome que exige publicar o morir y viene acompañado por pensamientos obsesivos, presión por el perfeccionamiento continuo, ansiedad por publicar y noches sin dormir (Cooper, 2012; Neill, 2008). Además, la vigilancia que implantan los sistemas de evaluación fuerza a aumentar continuamente la cantidad de trabajo. La cuantificación de las citas que ya permite Google Scholar, la enseñanza evaluada por los estudiantes, los sistemas nacionales de acreditación y otras métricas cada vez más imperativas transforman al académico en un administrador de sí mismo, cuyo desempeño debe ser constantemente monitorizado. El recurso actual al self-branding y a la autopromoción, como elementos fundamentales del impacto académico, sume al mundo universitario en un profundo conflicto ético sobre cuáles son los principales objetivos de la investigación contemporánea. Esto conduce a veces a un desgarro íntimo creado por la pugna interna sobre cómo establecer prioridades entre, por un lado, las exigencias que provienen de la gobernanza y, por otro, los valores personales.

Con su mordiente habitual, Mark Fisher (2014) usó la palabra desviado (sidetracked) para describir las consecuencias de estos procesos. La academia contemporánea, dijo hablando de sí mismo, te desvía de tus finalidades, te induce a estar sustituyendo lo que querrías hacer por lo que estás obligado a hacer. Esta tendencia desgarra al individuo continuamente forzándolo a desviarse de lo que se percibe como importante para cumplir con todas las exigencias institucionales de la gobernanza digital. Otra palabra clave para este proceso es dislocación (dislocation). Es el término usado por Jonathan Malesic (2016) en un artículo publicado en Times of Higher Education titulado emblemáticamente The 4o-Year-Old Burnout. Why I gave up tenure for a yet-to-be-determined career, donde se recoge una de las muchas cartas de despedida publicadas por académicos que han abandonado la universidad en los últimos años. Malesic retoma 
la palabra dislocation de Christina Maslach (2003), una psicóloga de Berkeley especialista durante años en el análisis del agotamiento laboral (burnout). En su libro The truth about burnout, Maslach y Leiter (1997) afirmaban que el agotamiento es precisamente el indicador del daño que se produce entre el ser de las personas y el deber ser que impone su trabajo. La carrera académica está expuesta al burnout de una manera amplificada, ya que, en la evaluación del rendimiento académico que se exige a los profesores, las prioridades e intereses de estos no coinciden con los criterios impuestos por la gobernanza académica. Cuando este daño se vuelve crónico, como afirma Malesic (2016), surgen las tres características principales del burnout: agotamiento, cinismo e ineficacia profesional. Por tanto, no es sorprendente descubrir los alarmantes datos que se han recogido al principio de este texto relativos al aumento del burnout en el entorno académico (Guthrie et al., 2017). Por añadidura, a este conflicto de prioridades entre el profesor como persona y las exigencias que le lanza la gobernanza académica, en muchas ocasiones, el primero trata de compensar la distancia entre sus prioridades personales y sus obligaciones institucionales mediante una carga de trabajo adicional para responder a ambas. Si a todo esto le sumamos la precariedad económica y material que domina el mundo de la investigación, la academia contemporánea se presenta, antológicamente, como una de las fábricas de enfermedad más emblemáticas. Rosalind Gill (2015) ha hablado de «heridas ocultas de la academia neoliberal» para describir la presión cada vez mayor y la reducción continua de la autonomía entre los académicos. Hay que señalar que, conforme pasa el tiempo y estas heridas se hacen más evidentes, están comenzando a reconocerse como problemas dentro y fuera de la universidad. El desgarro entre el estrés organizacional y valores personales produce conflictos éticos particularmente graves entre los profesores más motivados por un sentido de justicia social (Malesic, 2016; Maslach, 2003). En algunos casos, como hemos visto anteriormente, esta divergencia se vuelve insostenible y estamos asistiendo a un proceso de renuncias a proseguir la carrera universitaria por parte de académicos en estos últimos años (Coin, 2017). 


\section{LA ACADEMIA ENFERMA}

Los datos sobre las consecuencias de todos estos procesos en los últimos años se multiplican. En diferentes investigaciones y congresos, se ha descrito la existencia de una verdadera crisis de salud mental en la universidad contemporánea. No solo afecta a los profesores, también entre los estudiantes, la precariedad y la cuestión de la deuda demuestra que, incluso antes de entrar en las aulas y considerar las condiciones materiales de la docencia, la enseñanza universitaria causa ya problemas que se reflejan en su salud mental (Toscano et al., 2014).

La American College Health Association realiza una encuesta anual sobre la salud mental de los estudiantes estadounidenses entre los que encontramos datos alarmantes. La encuesta del 2015, por ejemplo, reveló que el $20 \%$ de los estudiantes fueron diagnosticados con depresión a lo largo de su vida, y que el $13 \%$ y el $16 \%$, respectivamente, habían sido tratados por depresión y ansiedad en los últimos 12 meses. El 7 \% sufrieron ataques de pánico en ese año. El $86 \%$ afirmaron sentirse sobrecargados, emocionalmente exhaustos $(82 \%)$, muy tristes $(64 \%)$, muy solos $(59 \%)$, sin esperanza $(48 \%)$, llenos de ira $(38 \%)$ o con sobrecarga de ansiedad (57\%). Alrededor de un tercio de los estudiantes (35\%) dijeron que se sentían tan deprimidos que era imposible cumplir con su trabajo (American College Health Association, 2015). También en 2015, otro estudio, Healthy Minds Study, realizado por la Healthy Minds Network (2016), basado en una muestra de 16 ooo estudiantes americanos, reveló que alrededor del $35 \%$ de ellos contabilizaba al menos un criterio de trastorno mental. Un $20 \%$ de los estudiantes tenía síntomas de depresión media y $12 \%$ de depresión intensa en ese mismo año (Healthy Minds Network, citado en The Provost's Committee on Student Mental Health, 2016).

En opinión de Richard Kadison, jefe de los servicios de salud mental de la Universidad de Harvard, la crisis de salud mental en las universidades se ha visto influida por el creciente enfoque económico vinculado a la educación secundaria, la participación excesiva en actividades extracurriculares y la atención exagerada a los resultados académicos. En términos longitudinales, el informe incluso sugiere que el estudiante promedio en el 2000 tenía el mismo nivel de ansiedad que un paciente psiquiátrico en 1950 (Henriques, 2014). Estos datos provienen de los Estados Unidos, donde el alto coste de la educación universitaria hace más vulnerables a los alumnos por el miedo al fracaso. Este mismo fenómeno se ha verificado en el Reino Unido, donde se ha informado de un aumento del $28 \%$ en las consultas de los servicios de salud mental, aso- 
ciado a la propagación del temor económico, la ansiedad y la depresión derivados del aumento de las tasas de matrícula desde finales de la década del 2000 (Gani, 2016). Según este autor, el precio de la matrícula, la deuda, el mercado laboral y el miedo a fracasar son factores determinantes para explicar la ansiedad y la depresión. La relación entre la depresión, la percepción de inadecuación y la competencia neoliberal vuelve aquí a manifestarse, como escribió Mark Fisher (2014), en una interpretación que vincula una vez más los orígenes de la depresión no con un trauma familiar o un desequilibrio químico, sino con un contexto social problemático en el cual el individuo está en el lado equivocado del poder social. Como ha declarado Shelly Asquith, vicepresidenta de la National Union of Students (NUS), uno de los sindicatos de estudiantes ingleses:

The value of education has moved away from societal value to «value for money» and the emphasis on students competing against each other is causing isolation, stress and anxiety (Gani, 2016)

Además, según esta misma fuente, se ha puesto a competir a las universidades y a gastar mucho en publicidad para captar financiación. La evidencia es cada vez más clara: la mercantilización de la educación tiene un impacto considerable en la salud mental de los estudiantes.

Por si esto no fuese ya suficientemente preocupante, hay que mencionar los datos más graves que afectan a los estudiantes de doctorado. La investigación de Levecque et al. (2017), realizada sobre una muestra de 3659 doctorandos belgas, destaca cómo el estrés y el malestar crecen a la par que la inseguridad laboral, el aumento de la carga de trabajo, el creciente desequilibrio entre la demanda y la oferta de empleos, los recortes de la financiación pública, la competencia académica y el crecimiento de contratos precarios. Por todos estos motivos, informa el estudio, los estudiantes de doctorado reportan testimonios de depresión, ansiedad y agotamiento físico y emocional que se concretan en que cerca de un tercio de la muestra presentaba una situación de riesgo de trastorno psiquiátrico y riesgo de depresión en uno de cada dos estudiantes. 


\section{CONCLUSIONES: SABOTEAR AL EVALUADOR}

A tenor de los datos expuestos, surge la pregunta de hasta qué punto vale la pena mantener una gobernanza universitaria tan profundamente incapacitante que perturba hasta la salud mental de profesores y estudiantes. Refiriéndonos exclusivamente a la evaluación y la supervisión continua del rendimiento que hemos abordado a lo largo del artículo, muchos expertos en política científica han destacado en los últimos años que el análisis de costos y beneficios de la evaluación en la universidad no ofrece, ni mucho menos, una correlación clara que favorezca la pertinencia de los actuales modelos de evaluación. De hecho, cabe preguntarse si los costos económicos de dichos procesos de evaluación son mayores que los ahorros que permiten. En Italia, quien se ha ocupado más rigurosamente de este tema en relación con el ejercicio de evaluación nacional es Alberto Baccini (2010). En un trabajo reciente, Baccini (2017) ha observado que, aunque el sentido común da por hecho que la evaluación del desempeño es necesaria porque permite «distribuir la financiación universitaria de forma meritocrática», en su opinión, esta afirmación debería analizarse críticamente. Por lo general, los costes del modelo son tan gravosos que ponen en cuestión los beneficios que pueda proporcionar a la ciencia y a la universidad (Baccini, Coin y Sirilli, 2013).

En los últimos años, diversos análisis han puesto de manifiesto las consecuencias potencialmente perjudiciales de la universidad neoliberal (The Economist, 2010; Shaw y Ward, 2014; Philips y Heywood-Roos, 2015). En Publish or perish, but at what cost?, Ushma S. Neill (2008) mostró cómo la presión cuantitativa para publicar resultados y aumentar la productividad no solo pone en peligro potencialmente la salud del personal académico, sino que hace aumentar la vulnerabilidad de la investigación hacia prácticas fraudulentas tales como el plagio, el autoplagio, la falsificación de datos o el uso de ghost-writers. En el 2011, Douglas N. Arnold y Kristine K. Fowler hablaron de «números perversos» (nefarius numbers) para describir los trucos utilizados por la comunidad científica internacional para escalar en los rankings de bibliometría. Cuando las métricas se transforman en fines en sí mismos, la ciencia se ve inmediatamente expuesta a comportamientos oportunistas, como el nacimiento de «alegres cuadrillas de academicos entusiastas que se citan mutuamente» como las denominó Alessandro Figà-Talamanca (2000). Incluso sin adentrarnos en las complejas dimensiones afectivas de la evaluación que aquí hemos esbozado, tal vez sea hora de reconocer con Baccini (2017) que «hemos adoptado un modelo cuyos beneficios desconocemos, pero que es muy caro». Por ahora, sin 
duda, «brinda un gran servicio a la política», que puede justificar mediante la evaluación decisiones discrecionales de recortes y distribución de recursos ajustados a la retórica tecnocrática de objetividad de los números.

Quizá ha llegado el momento de reconsiderar colectivamente los objetivos de la producción intelectual y de la supervisión-evaluación digital, porque más que un ahorro que permite que los fondos universitarios se distribuyan de manera meritocrática, o más que un modo de detectar a los incompetentes, la evaluación parece estar legitimando un programa de recortes y una reformulación de las finalidades de la universidad, lo que debería despertar nuestra indignación colectiva.

\section{BIBLIOGRAFÍA}

Abravanel Roger (2008). Meritocrazia. Milano: Garzanti.

American College Health Association (2015). American College Health Association-National College Health. Assessment II. Thompson Rivers University Executive Summary. Hanover, MD: American College Health Association.

Arnold, Douglas N. y Fowler, Kristine K. (2011). Nefarious numbers. Notices of the American Mathematical Society, 58(3), 434-437.

Arrow, Kenneth J. (1973). Higher education as a filter. Journal of Public Economics, 2(3), 193-216.

Autor, David H., Katz, Lawrence F. y Kearney, Melissa S. (2006). The polarization of the U.S. labor market. American Economic Review, 96(2), 189-194.

Baccini, Alberto (2010). Valutare la ricerca scientifica. Uso e abuso degli indicatori bibliometrici. Milano: Il Mulino.

Baccini, Alberto (2017). Valutazione, i dubbi prima del «tagliando, Scuolaz4. Recuperado de: http://www.scuola24.ilsole24ore.com/art/universita-ericerca/2017-08-22/valutazione-dubbi-prima-tagliando180oo3.php?uuid=AEUoHBGC [Consultado el 23 de marzo de 2018]. 
Baccini, Alberto, Coin, Francesca y Sirilli, Giorgio (2013). Costi e benefici della valutazione della ricerca e della didattica. Paradoxa Forum, VII-2, 49-61.

Burdett, Kenneth (1978). The testing and sorting functions of higher education. Journal of Public Economics, 10(1), 117-122.

Coin, Francesca (2012). La valutazione: da ranking a profilo di qualità. Scuola Democratica, 5, 160-165.

Coin, Francesca (2017). On quitting. Ephemera. Journal of theory and politics in organisation, $17(3), 235^{-249}$.

Cooper, Mark J. (2012). Antidotes for the publish or perish syndrome. Recuperado de: https://www.facultyfocus.com/articles/facultydevelopment/antidotes-for-the-publish-or-perish-syndrome [Consultado el 24 de marzo de 2018].

Dangarembga, Tsitsi (1988). Nervous conditions. London: Women's Press.

Else, Holly (2017). Academics face higher mental health risk than other professions. Recuperado de: https://www.timeshighereducation.com/news/academics-facehigher-mental-health-risk-than-other-professions [Consultado el 25 de marzo de 2018].

Figà-Talamanca, Alessandro (2000). L'impact factor nella valutazione della ricerca. Return on Academic Research. Recuperado de: http://siba.unipv.it/biblioteche/info/SISSCOWEB_A_Fig_Talama nca_L\%27impact_factor_nella_valutazione_della_ricerca_SISSCO WEB.htm [Consultado el 25 de marzo de 2018].

Fisher, Mark (2014). Good For Nothing. The Occupied Times. Recuperado de: https://theoccupiedtimes.org/? $\mathrm{p}=12841$ [Consultado el 26 de marzo de 2018].

Gani, Aisha (2016). Tuition fees have led to surge in students seeking counselling. Recuperado de: https://www.theguardian.com/education/2016/mar/13/tuitionfees-have-led-to-surge-in-students-seeking-counselling [Consultado el 23 de marzo de 2018]. 
Garfield, Eugene (2006). Commentary: fifty years of citation indexing. International Journal of Epidemiology, 35, 1127-1128. doi: 10.1093/ije/dyligo.

Gill, Rosalind (2015) Rompiendo el silencio: las heridas ocultas de la universidad neoliberal. Arxius de Ciéncies Socials, 32, 45-58.

Guthrie, Sussan, Lichten, Catherine, Van Belle, Janna, Ball, Sarah, Knack, Anna y Hofman, Joanna (2017). Understanding mental health in the research environment. A Rapid Evidence Assessment. Santa Monica: RAND Corporation.

Hall, Gary (2016). The Uberfication of the University. Minnesota: University of Minnesota Press.

Henriques, Gregg (15 de febrero, 2014). College Student Mental Health Crisis. Psicology today. Recuperado de: https://www.psychologytoday.com/blog/theoryknowledge/201402/the-college-student-mental-health-crisis [Consultado el 23 de marzo de 2018].

Hirtt, Nico (2010). In Europa, le competenze contro i saperi. Le Monde Diplomatique. Recuperado de: http://reteeco.it/2011/documenti/35-riflessioni/17356-in-europa-lecompetenze-contro-i-saperi.html [Consultado el 20 de marzo de 2018].

Kostoris Padoa Schioppa, Fiorella (2012). Ragioni, criteri e modalita' di realizzazione di un test sulle competenze effettive di carattere generalista dei laureandi italiani, ANVUR. Recuperado de: https://www.uniba.it/organizzazione/amministrazione/dirgen/cso /area-percorsi-qualita/VQAF/Kost.pdf [Consultado el 24 de marzo de 2018].

Levecque, Katia, Anseel, Frederik, De Beuckelaer, Alain, Van der Heyden, Johan y Gislef, Lydia (2017). Work organization and mental health problems in $\mathrm{PhD}$ students. Research Policy, 46(4), 868-879. doi: 10.1016/j.respol.2017.02.0o8.

Lupton, Deborah (2016). The quantified self. A sociology of self-tracking. Malden: Polity Press. 
Lupton, Deborah, Mewburn, Inger y Thomson, Pat (2017). The Digital Academic: Critical Perspectives on Digital Technologies in Higher Education. New York: Routledge.

Marginson, Simon (2009). The knowledge economy and higher education: A system for regulating the value of knowledge. Higher Education Management and Policy, 21(1), 31-46.

Malesic, Jonathan (2016) The 40-Year-Old Burnout. Why I gave up tenure for a yet-to-be-determined career. The Chronicle of Higher Education. Recuperado de: http://www.chronicle.com/article/The40-Year-Old-Burnout/237979 [Consultado del 24 de marzo de 2018].

Maslach, Christina (2003). Burnout. The Cost of Caring. Los Altos: Malor Book.

Maslach, Christina y Leiter, Michael P. (1997). The truth about burnout: How organizations cause personal stress and what to do about it. San Francisco: Jossey-Bass.

Moore, Phoebe V. (2017). The Quantified Self in Precarity: Work, Technology and What Counts. New York: Routledge.

Neill, Ushma S. (2008). Publish or perish, but at what cost? Journal of Clinical Investigation 118(7), 2368. doi: 10.1172/JCI36371.

Phillips, Siobhan y Heywood-Roos, Rhona (2015). Job security for early career researchers is a significant factor in helping research make an impact. Impact of Social Sciences Blog. Recuperado de: http://blogs.lse.ac.uk/impactofsocialsciences/2015/o6/3o/earlycareer-paths-of-doctorate-holders-esf-pilot/ [Consultado el 20 de diciembre de 2017].

Sassen, Saskia (2015). Espulsioni. Brutalità e complessità nell'economia globale. Roma: Il Mulino.

Scelfo, Julie (27 de julio, 2015). Suicide on Campus and the Pressure of Perfection. The New York Times. Recuperado de: https://www.nytimes.com/2015/o8/o2/education/edlife/stresssocial-media-and-suicide-on-campus.html?mcubz=o [Consultado el 20 de marzo de 2018]. 
Shaw, Claire y Ward, Lucy (2014). Dark thoughts: why mental illness is on the rise in academia. The Guardian, 6 de marzo. Recuperado de: http://www.theguardian.com/higher-educationnetwork $/ 2014 / \mathrm{mar} / \mathrm{o6} / \mathrm{mental}$-health-academics-growingproblem-pressure-university [Consultado el 12 de marzo de 2018].

Striphas, Ted (2015). Algorithmic culture. European Journal of Cultural Studies, $18(4-5)$, 395-412.

The Economist (2010). The disposable academic. Why doing a $\mathrm{PhD}$ is often a waste of time. The Economist (Christmas Special, 16 de diciembre). Recuperado http://www.economist.com/node/17723223?story [Consultado el 22 de marzo de 2018].

The Provost's Committee on Student Mental Health (2016). The State of Student Mental Health on College and University Campuses. Recuperado de: http://www.mentalhealth.umn.edu/download/Provost_s_Committ ee_on_Student_Mental_Health_White_Paper_Final.pdf [Consultado el 22 de marzo de 2018].

Toscano, Emanuele (Coord.) (2014). Ricercarsi. Indagine sui percorsi di vita e lavoro del precariato universitario. Recuperado de: https://www.academia.edu/11162215/Ricercarsi._Indagine_sui_perc orsi_di_vita_e_lavoro_del_precariato_universitario [Consultado el 22 de febrero de 2019]. 